\title{
PAPEL DE LA EDUCACIÓN EN LA PRIMERA INFANCIA EN UNA SOCIEDAD DE CAMBIO
}

\author{
Esther Sánchez Morales \\ Licenciada en Psicopedagogía \\ Universidad de Granada \\ sthersm@correo.ugr.es
}

Recepción Artículo: 26 junio 2020

Admisión Evaluación: 15 octubre 2020

Informe Evaluador 1: 25 noviembre 2020

Informe Evaluador 2: 23 noviembre 2020

Aprobación Publicación: 27 noviembre 2020

\section{RESUMEN}

El presente estudio de investigación pretende indagar en el problema actual en el que está in- mersa la sociedad en lo referente a cuestiones de educación. La sociedad en la que vivimos está sometida a una serie de cambios sociales, culturales, económicos y educativos que se ven afec- tados por la globalización. La educación busca inculcar normas y comportamientos que sirvan de herramientas para el desarrollo personal y social de las nuevas generaciones. A su vez el papel de los padres en el desarrollo de sus hijos y la manera de vincularse a ellos, hace de su labor algo esencial en la vida, pero el mundo laboral, la poca disponibilidad, la falta de herramientas para actuar en la educación de sus hijos, hace que el proceso sea más complejo, sobre- todo en la primera infancia. En este artículo se presenta la educación como valor fundamental en la sociedad y los cambios asociados al nacimiento de nuevas generaciones.

Palabras clave: transformaciones socioculturales; educación; educación infantil; familia; estimulación temprana

\section{ABSTRACT \\ Role of education in early childhood in a society of change. This research study aims to investigate the current problem in which society is immersed in matters of education. The society in which we live is submit- ted in a series of social, cultural, economic and educational changes that are affected by globalization. Education seeks to instill standards and behaviors that serve as tools for the personal and social development of new gene- rations. At the same time, the role of parents in the development of their children and the way to link to them makes their work essential in life, but the world of work, lack of availabi- lity, lack of tools to act in education Of their children makes the process more complex, espe- cially in early childhood. This article presents education as a fundamental value in society and the changes associated with the birth of new generations.}

Keywords: Sociocultural transformations; education; early childhood education; family; early stimulation 


\section{PAPEL DE LA EDUCACIÓN EN LA PRIMERA INFANCIA EN UNA SOCIEDAD DE CAMBIO}

\section{OBJETIVOS DEL ESTUDIO}

- Indagar en las posibles causas del problema actual en el que está inmersa la educación del siglo XXI.

- Analizar influencia y cambios en las familias, variaciones a nivel social, político, educativo, personal, en valores, económico, etc.

- Estudiar los estilos educativos que influyen en el modelo familiar y por consiguiente en el comportamiento social y afectivo de los alumnos, en las primeras etapas.

Reconocer la importancia actual de la educación en la primera infancia y el trabajo de la estimulación temprana.

\section{INTRODUCCIÓN}

\section{Una sociedad en constante cambio}

Cualquier persona, que mire a la sociedad puede percibir que ha sufrido un gran cambio en las últimas décadas a nivel social, personal, económico, educativo y de valores. Cambios en el desarrollo del siglo XXI que han generado un fenómeno de globalización en diferentes contex- tos y en especial en el de la familia. La sociedad está sumergida en un proceso de modificacio- nes que afectan al sistema educativo y al significado verdadero de la educación. Todo esto ha llevado a la existencia de una gran preocupación por la infancia, Ilevando a cabo cambios a nivel legislativo, escolar, familiar y modelos de crianza que se van adaptando a estilos más permisivos que antes.

Estos cambios a distintos niveles están produciendo una falta de conocimiento, miedos de las familias al no saber cómo actuar y una ligada pérdida de control educativo en los centros esco- lares y familiares.

Las sociedades rápidamente cambiantes se enfrentan a desafíos en los que se intenta equilibrar valores tradicionales familiares que buscan la armonía con valores emergentes que promueven la autonomía. Es difícil para los padres inculcar una adaptación a los nuevos entornos sociales, especialmente en las sociedades que se están modificando (Dasen, 2000).

Todo este cambio llevo asociado, como señala el Centro de Investigaciones Sociológicas (2005), el nacimiento de comportamientos inadecuados, faltas de control y actuaciones vandá- licas relacionadas con el maltrato de los hijos a padres, a abuelos y entre iguales que demues- tran que existen carencias en la educación.

La transmisión de la educación se vuelve más compleja para las familias cuando se lleva a cabo en tiempos de cambios en la sociedad, ya que los padres están perdidos en el conocimiento, adquisición de nuevos valores e intentando mantener los del pasado. Esta revisión se centra en las dificultades y nuevos retos de las familias sumergidas en estos cambios, culturales y de desarrollo.

Esto nos lleva a hacernos diversos interrogantes: ¿Cómo se está educando? ¿Sabemos afron- trarlo? ¿Qué papel se le está dando a la educación hoy en las familias? ¿Está la familia adaptada a los cambios de una sociedad del siglo XXI?

\section{Variaciones culturales}

Se han llevado a cabo importantes transformaciones en la cultura (Fisek y amp; Kagitc ibasi, 1999) la industrialización, la urbanización, el aumento de las oportunidades educativas, y el aumento de empleo para las mujeres que afectan cada día a los valores de la familia y la es- tructura.

\section{Variaciones políticas}

Las decisiones y actitudes políticas afectan a la vida familiar, los roles de género, los derechos y las responsabilidades, las actividades diarias y la comunicación. Las personas con actitudes políticas conservadoras enseñan diferentes valores a sus hijos que sus contrarios con ideologías más liberales. Algunas actitudes dan prioridad a la fe religiosa y la obediencia, mientras que en otras dan prioridad a la tolerancia (Pew Research Center, 2014). 


\section{Variaciones en la transmisión de valores}

Muchos padres experimentan un dilema acerca de cómo llegar a un equilibrio entre los valores tradicionales y los modernos, mientras transmiten valores a sus hijos (Akyll et al., 2014).

A pesar de los cambios significativos, existen algunos valores básicos que deben permanecer estables en el tiempo. El individualismo en los comportamientos de los niños pueden llevar al desarrollo de valores que alejen de la lealtad a la familia. De cualquier modo, las formas que expresan esta lealtad pueden ser bastante diferentes a las de sus padres. Ayudando a las familias a llegar a un acuerdo se puede aliviar a los progenitores que están preocupados. Esto lleva asociada una mejora en la falta de modales si el niño ha interiorizado el valor del respeto.

\section{Variaciones en la Educación}

Uno de los grandes cambios está siendo la igualdad entre padres e hijos. Como comentan Alegret, Comelas, Font y Funes, (2005); Cowan, Powell y Cowan, (1998); López, (1995) , partimos de unos fundamentos estandarizados que gozan de prestigio, tales como que hay que ser "amigos" de los hijos, no se deberían imponer cosas sino llegar a un consenso entre ambas partes, y buscar la otra opción de educación como puede ser la democrática.

Otro cambio relevante, según Beck y Beck- Gernsheim, (2001), está en el mundo laboral y la escasa disponibilidad para los cuidados de los hijos. En esta línea, el factor tiempo de las familias implica modificaciones notables en la sociedad en la que nos vemos inmersos. Existe una exigencia por la formación, la adquisición de títulos que aseguran un bienestar económico y social, y pueden ayudar a la estabilidad familiar y económica.

Todo esto, lleva consigo retrasar los compromisos de pareja, la edad de formar una familia y por ende ganas y empeño por sacarla adelante. Pero a su vez, el factor tiempo dedicado a las mismas se ve influenciado. Estas modificaciones llevan de la mano aspectos positivos como una riqueza de conocimiento y diversidad en las formas de vida. Actualmente, vivimos en una sociedad globalizadora y consumista. En línea con Gubbins y Berger, (2004), el escaso tiempo que dedican los padres a las familias, crea ciertas carencias afectivas que intentan compensar con objetos materiales, donde el consumismo adquiere su valor y comienzan a ser más permi- sivos y flexibles de la cuenta, lo que nos Ileva a alejarnos de un adecuado modelo de crianza que dé valor a la vida familiar, como señalan Montesinos, (2002); Jiménez, (2005).

Otro aspecto de gran relevancia es la influencia en la familia del trabajo llevado a cabo por ambos progenitores.

En la actualidad, el papel del hombre y la mujer van en el mismo sentido tanto profesional como familiar, lo que conlleva un bienestar personal para ambos.

Las ayudas para el cuidado diario de los hijos, la necesidad de la experiencia a la hora de tomar decisiones se han visto modificadas en las últimas décadas a medida que más mujeres han comenzado a trabajar fuera del hogar, el cuidado y educación de los hijos ha sido un tema relevante para las familias (Claessens, 2012).

En línea con Coloma y Forni, (2007), se dedica menos tiempo a la familia, por lo que nacen una serie de carencias en muchos ámbitos del desarrollo de los hijos. A su vez, como señalan Covarrubias, Mufioz y Reyes, (1983); Raczynski y Serrano, (1986) , la inserción de la mujer en el mundo del trabajo ha podido influenciar este cambio, conllevando un descenso en la natalidad (Donoso, 2007) que se ve influenciado en el trato de los hijos que se tienen.

Otro factor positivo relacionado, según Bornstein (2002) es que los padres altamente educados hablan más con sus hijos, utilizan menos métodos con una severa disciplina, dedican más tiempo a la enseñanza y a proporcionar entornos más estimulantes desde la infancia hasta la adolescencia. Según Wilson, (1984) y Gassama, (2012), a su vez tienen influencia los niveles económicos de las familias en el crecimiento de la inteligencia de los mismos.

El proceso de enseñanza y aprendizaje va paralelo a la evolución de la civilización humana. Actualmente estamos en una nueva evolución, como desarrollo de las Tecnologías de la Infor- mación y las Comunicaciones (TIC), 


\section{PAPEL DE LA EDUCACIÓN EN LA PRIMERA INFANCIA EN UNA SOCIEDAD DE CAMBIO}

el uso de las redes sociales que afecta a la realidad edu- cativa. El conocimiento llega en mayor medida gracias al Internet y es gracias a la educación por lo que se busca adaptarse a los cambios a lo largo de la vida (Cobo y Moravec, 2011).

Todos estos cambios se ven en gran parte influidos Hoy por el aumento y desarrollo de las comunicaciones y con ende la tarea socializadora que era exclusiva de las familias, y que hoy es compartida con los medios de comunicación a diario. (Rodrigo y Palacios, 1998).

Se debe destacar, al igual que comentan Palomares, Garrote, Serrano y López (2012) que se hace indispensable la participación, colaboración y concienciación de las familias en el centro educativo, siendo la educación herramienta fundamental en la sociedad de hoy y de siempre.

\section{Papel crucial de la educación como herramienta de ayuda en estos cambios}

Existe una imperiosa necesidad de formar a los hijos y alumnos en la adaptación a las exigen- cias del mundo en el que vivimos. (Youniss, 1994).

La educación es el proceso mediante el que el ser humano consigue las diferentes herramientas para su desarrollo en la sociedad y su realización personal. Como señala Hernández (2003) es el proceso mediante el cuál se ejerce una determinada influencia sobre la "nueva generación" con el propósito consciente o no de inculcarle una serie de normas, valores y comportamientos que les permita a todos y cada uno de sus miembros realizar los roles sociales para los cuáles han sido formados individual y colectivamente.

La educación debe adaptarse a estas modificaciones, desarrollando en el alumnado una serie de competencias que amplíen su abanico de actuación en diferentes situaciones de la vida, haciéndoles cada vez más competentes ante el respeto y la adversidad.

Por ello la importancia de analizar diferentes factores sociales, económicos, tipos de familias que puedan afectar a problemas emocionales y conductuales del alumnado, tomando como base la comunicación directa entre familia y escuela para poder intervenir en la problemática situa- ción actual con la educación. Pero no sólo desde la educación en el colegio se consigue adaptar un niño a la sociedad.

El desarrollo de la persona y en concreto el desarrollo del niño necesita del estudio de diversos factores: hereditarios, genéticos. socioculturales, etc. El papel de los padres en el desarrollo de sus hijos y la manera de vincularse a ellos hace de su labor algo esencial en la vida.

La manera que tienen los progenitores de desarrollar y/o mantener las conductas de sus hijos, afecta al comportamiento de los mismos. Las acciones cotidianas se convierten en practicas de crianza que pueden ser adecuadas o conllevar futuros desórdenes en la falta de disciplina.

Como señalan, Chang y Dodge (2003), Eisenberg, Fabes-guthrie, Losoya, Murphy, Padgett y Poulin, (2001) Reiser, Shepard, y Stoolmiller, (2001), los procesos de aprendizaje en la infan- cia están influenciados por la familia y las personas que rodean a los niños, estos son estimu- lantes para su desarrollo y adquisición de hábitos que le permiten desenvolverse en la vida e interactuar en los contextos sociales, familiares y escolares. Por ello, el proceso de la educación es crucial desde las primeras edades.

\section{En busca del mejor estilo de crianza}

Se habla, se comparte, se empatiza, se dialoga, pero luego se vuelve al modelo autoritario que busca imponer criterios de forma exigida poniendo sus límites. Por otro lado, las familias, tam- bién conllevan cambios positivos como se señala en la teoría de Bronfenbrenner (1987), la familia busca vías de encuentro bidireccionales, que se caracterizan por la presencia de un acercamiento físico directo y un clima afectivo entre sus miembros.

Por otro lado el valor psicológico de los niños, es decir, el valor de los vínculos afectivos, ha cobrado importancia, Io que ha dado lugar a estrategias de crianza democráticas. Estas incluyen el uso de recompensas y razonamientos, que apoyan la independencia en lugar del control au- toritario y, a su vez, admite la obediencia (Kagıtcbas, 1996; Sunar, 2002). 
Los padres hoy día, tienden cada vez más a valorar la autonomía de sus hijos, pero a cambio, ellos sienten que están perdiendo su capacidad para protegerlos.

Algunas veces, utilizan estrategias democráticas de crianza, y otras, cuando se enfrentan a una situación estresante, lo que les lleva hacia el otro extremo del autoritarismo.

\section{Valor de los vínculos afectivos como ayuda}

La expresión de las emociones positivas, como el amor, la felicidad y la alegría, ha sido fo- mentado con la educación emocional en las aulas.

Desde la infancia hasta la edad adulta, las madres utilizan el afecto físico: besos y abrazos. Con las actuaciones de la vida diaria de los progenitores se expresan actos de amor. Los padres generalmente están más distantes, y expresan su amor o cuidado dedicándoles un tiempo de calidad.

\section{Nacimiento de la importancia de la etapa de Educación Infantil: estimulación temprana.}

El desarrollo del cerebro en el niño en la primera infancia es vital y depende del ambiente en el que el niño se desarrolle. Hace poco tiempo, los científicos de la neurociencia decían que desde el momento en el que el niño nacía, la estructura del cerebro estaba genéticamente esta- blecida. No se daba valor a que las experiencias en los primeros años de vida tenían una im- portante repercusión en el cerebro y su posterior desarrollo para la vida, en línea con Shore (1997).

La Educación Infantil se valora cada vez más como una necesidad imperiosa de la vida mo- derna. Por ello, algunos padres consideran esencial el trabajo de la etapa de Infantil. Tao (2012) señaló la eficacia de la educación de la primera infancia entorno al aprendizaje de las conduc- tas y cognitivo.

Jakes y Debord (2010) investigaron el efecto ambiental de la familia, la escuela y la comunidad. Señalan que los niños y los jóvenes están muy influenciadas por la participación de la familia en la edad temprana, pero a medida que aumenta la edad, esta contribución se vuelve menos destacable. En la primera etapa de Educación Infantil la conexión entre las familias y el centro educativo es de gran valor. Suelen ser más permisivos en las conductas de sus hijos que en etapas posteriores, donde existe menos seguridad en la educación de sus hijos. Esto puede llevar a serios problemas en la primeros años de vida, ya que la falta de control lleva a estable- cer conductas inadecuadas, por no haber puesto límites en el debido momento.

En esta importante etapa, con una gran repercusión en el desarrollo posterior, padres y madres intentan educar a sus hijos por primera vez con prácticas educativas caracterizadas por el con- trol y las exigencias, que inciden en la existencia o no de normas y disciplina. Otras dimensio- nes presentes son el afecto y la comunicación, caracterizadas por el grado de apoyo y afecto explícito hacia los hijos y la mayor o menor comunicación entre padres e hijos (Vega, 2006). Esta experiencia de control y afecto influye directamente en la dinámica familiar, y genera una sensación de satisfacción o insatisfacción de necesidades básicas de comunicación y afecto. La

calidad de vida se entiende como un concepto que refleja las condiciones deseadas por una persona respecto a las dimensiones de la vida relacionadas con el bienestar emocional, las re- laciones interpersonales, el bienestar material, el desarrollo personal, el bienestar físico, la au- todeterminación, la inclusión social y los derechos (Córdoba, Gómez y Verdugo, 2008).

Por ello, la participación de la familia y de la comunidad son factores protectores del desarrollo de los hijos. Así pues, parece que la intervención temprana es una cuestión clave y tiene un efecto positivo sobre el rendimiento de la inteligencia de los niños.

La educación se ve en la necesidad de llevar a cabo enseñanzas con métodos avanzados e in- novadores, programas con ajustes en la educación de la primera infancia y que vayan destina- das también a niños de bajos estratos socioeconómicos (Zill, Moore, Smith Steif, y Coiro, 1995). Por lo tanto, diversos tipos de programas de intervención han sido diseñados y realiza- dos con éxito para compensar las profundas dificultades de niños procedentes de familias eco- nómicamente en desventaja. 


\section{PAPEL DE LA EDUCACIÓN EN LA PRIMERA INFANCIA EN UNA SOCIEDAD DE CAMBIO}

Se deben buscar programas de enriquecimiento, en los que los padres y las escuelas hagan actividades organizadas para que estén involucrados en actividades escolares y aumentar así los vínculos sociales con las escuelas. Estos programas son eficaces para las familias minori- tarias que están aislados o tienen muy pocos lazos sociales con las escuelas.

Los niños de los países más pobres y de un nivel socioeconómico inferior debe dar el privilegio de asistir a estos programas (Fernald, Weber y Ratsifandrihamanana, 2011).

La pobreza es un fenómeno bien conocido, y la educación también es para desfavorecidos. Por ello, no se debe olvidar la importancia de los primeros años de vida de los niños. La pobreza y la cultura que rodea a los niños pequeños tienen importantes efectos permanentes sobre la sa- lud, el desarrollo social y cognitivo. Los programas de intervención deben identificar a los niños desfavorecidos a una edad temprana y trabajar con ellos.

\section{CONCLUSIONES}

Son muchos los cambios a los que se somete la sociedad hoy día, pero el significado de la educación debe adaptarse a estas modificaciones.

La educación desde la primera infancia, con la prevención y estipulación temprana, se ve afec- tada por las transformaciones en las tecnologías y los nuevos avances que dan lugar a diferentes modelos educativos que llevan a la inestabilidad e inseguridad del profesorado y por ende a las familias. La ayuda a las familias en cuestiones educativas se encuentran en diferentes espacios pero el principal está en el centro educativo, por lo que si algo falla desde allí, todo se tambalea. La falta de unión entre familias y centros educativos, el indispensable valor de las familias para el desarrollo de los hijos como vínculo afectivo principal, el escaso tiempo dedicado a la educación y los nuevos cambios a los que no es fácil adaptarse por una inseguridad ante lo que vendrá, se interponen en el proceso.

Pero por otro lado, estas transformaciones desarrollan una sociedad, el cambio se abre al avance y propicia variaciones en las relaciones sociales, que nos acercan a la innovación tecnológica y de valores, estableciéndose el verdadero avance en la Educación. Los cambios rápidos en la sociedad generan un desafío para los padres de hoy día, por ello la educación debe ofrecer las herramientas para equilibrar las diferentes necesidades.

\section{REFERENCIAS BIBLIOGRÁFICAS}

Akyll, Y., Prouty, A., Blanchard, A., \& Lyness, K. (2014). Parents' experiences of intergenerational $\quad v$ a I u e trans- mission in Turkey's changing society: An interpretive phenomenological study. Journal of Family Psychother-apy, 25(1), $42-65$.

Alegret, J., Comellas, M. J., Font, P. \& Funes, J. (2005). Adolescentes: relaciones con los padres, drogas, sexualidad y culto al cuerpo. Barcelona: Grao.

Beck, U. \& Beck-Gernsheim, E. (2001). El normal caos del amor. Las nuevas formas de la relación amorosa (Trad. De D. Schmitz). Barcelona: Piadós. [V.0.: Das Ganz Normale Chaos Der Liebe. Frankfurt Am Main: Suhrkamp, 1990].

Bornstein, M.H. (2002). Handbook of parenting. Children and parenting. Yahweh, N.J.: Lawrence Erlbaum Associates.

Bronfenbrenner, U. (1987). La Ecología del desarrollo humano (1 Ed.). Barcelona: Paidós.

Bill, N.,Moore, K. A., Smith, E. W., Steif, T., \& Coiro, M.J. (1995). The life circumstances and development of children in welfare families: A profile based on national survey data. In P. Chase- Lansdale J. Brooks-Gunn (Eds.), Escape from poverty: What makes a difference for children? (pp.38-59). New York: Cambridge University Press.

Chang, S., \& Dodge, M. (2003). Parenting in relation of child emotion regulation and aggression. Journal of Family Psychology, 17 (4), 598-606.

CIS (2005). Opiniones y actitudes sobre la Infancia. Estudio 2621. Madrid: CIS. 
Claessens, A. (2012). Kindergarten child care experiences and child achievement and socioemotional skills. Early Childhood Research Quarterly, 27 (3), 365-375.

Cobo, C. y Moravec, j. W. (2011). Aprendizaje invisible. Hacia una nueva ecología de la educación. Barcelona: Colección Transmedia XXI.

Covarrubias, P, Muñoz, M, Reyes, C. (1983) ¿Crisis en la familia? Cuadernos del Instituto de Sociología. Pontificia Universidad Católica de Chile, Santiago.

Cowan, Ph. A., Powell, D. \& Cowan, C. P. (1998). Parenting Interventions: A Family Systems Perspective. En W. Damon, I. E. Digel \& K. A. Renninger (Eds.), Handbook of Child Psychology: Vol. (4), p. 3-72. Nueva York: Jonh Wiley.

Dasen, P. R. (2000). Rapid social change and the turmoil of adolescence: A cross-cultural perspective. Journal of Group Tensions, 29(1/2), 17-49.

Eisenberg, N., Losoya, S., Fabes, R.A.,Guthrie, I.K., Reiser, M.,Murphy, B.,Shepard, S.A., Poulin, R., \& Padgett, S.J. (2001). Parental socialization and Children's deregulated expression of emotion and externalizing problems. Journal of Family Psychology, 15(2), 183-205.

Fernald, L.Ch, Weber, A,Galasso, e. \& Ratsifandrihamanana, L. (2011). Socioeconomic gradients and child development in a very low income population: Evidence from Madagascar. Developmental Science, 14(4), 832847.

Fisek, G. O., \& Kagit"cibasi, C". (1999). Multiculturalism and psychotherapy: The Turkish case. En P. Pedersen (Ed.), Multiculturalism as a fourth force (pp. 75-90). Philadelphia: Taylor \& Francis.

Gassama, S. (2012). The correlation between poverty and learning: What can be done to help children with limited resources learn?

Gubbins, V. \& Berger, C. (2004). Pensar el desarrollo familiar. Una perspectiva transdisciplinaria. Centro de Estudios de la familia. Universidad Alberto Hurtado. Santiago. Recuperado el 21 Junio De 2012 En: http://Psicología.Uahurtado.Cl/ Vgubbins/?Page_ld=7

Hernández, Á. (2003). Introducción a la Ciencias de la Educación. Tercera edición.

Ediciones Uapa. Santiago de los Caballeros, República Dominicana.

Jakes,S. S., \& Debord, K. (2010). The interface of family, school, and community factors for the positive development of children and youth. Journal of Prevention \& Intervention in the Community. 38(3), 177-82.

Jiménez, A. B. (2005). Modelos y realidades de la familia actual. Madrid: Fundamentos.

Kagıtcıbası, C., Sunar, D., \& Bekman, S. (1988). Comprehensive preschool education project: Final report. Ottawa: International Development Research Centre.

López, F. (1995). Necesidades de la Infancia: respuesta familiar. Infancia y Sociedad, (30), 7-49.

Montesinos, R. (2002). Las Rutas de la masculinidad. Ensayo sobre el cambio Cultural y el mundo moderno. Barcelona: Gedisa.

Palomares, A., Garrote, D., Serrano, I. \& López, S. (2012). Educación Social y ciudadanía reflexiva y crítica. En Morales, S., Lirio, J. \& Marí, R. (Coord.), La Pedagogía Social en la Universidad. Investigación, Formación y Compromiso, (pp. 89-98). Valencia: Nau Llibres.

Pew Research Center (2014). Teaching the children: Sharp ideological differences, some common ground. Retrieved October 1, 2014, from http://www.people-press.org/2014/09/18/teaching-the-children-sharp-ideological-differences-some-common- ground/.

Rodrigo, M.J.; Palacios, J. (Coords.) (1998). Familia y desarrollo humano (p.501-519).

Madrid: Alianza Editorial.

Serrano, I., Ramos, A. \& Rodríguez, N. (1989). Política pública para la familia: Análisis Crítico de programas de servicio en Puerto Rico. Revista Interamericana de Psicología, (23), 103-118. 


\section{PAPEL DE LA EDUCACIÓN EN LA PRIMERA INFANCIA EN UNA SOCIEDAD DE CAMBIO}

Shore, R. (1997). Reconsiderando el cerebro: Nuevos Percepciones dentro del desarrollo temprano. New York, N. Y.: Instituto de las familias y el trabajo.

Stoolmiller, M. (2001). Synergistic Interaction of Child Mana- Geability Problems and Parents-Discipline Tactics in Predic- Ting Future Growth in Externalizing behavior for boys. developmental psychology. 37(6), 814-825.

Vega, M. (2006). Estilos de crianza y sus efectos. Taller para padres, Instituto Alemán de Valdivia. Recuperado el 06 Junio 2010, En: Http://www.Educarchile. CI Doi: 10.4067/ S0370-41062011000400004

Verdugo, M.; Córdoba, L. \& Gómez, J. (2005). Spanish version family quality of life survey.

Journal of Intellectual disability research, (49), 794-798.

Wilson, S.R. (1984). Risk and residence in early mental development, 795-805.

Youniss, J. (1994). Rearing children for society. In J. G. Smetana (Ed.), Beliefs about parenting: Origins and developmental implications (pp. 37-50). San Francisco, CA: Jossey-Bass 